\title{
Synonymous Logics: A Correction
}

\author{
Francis Jeffry Pelletier and Alasdair Urquhart
}

August 25, 2006

\section{Introduction}

In a paper devoted to problems of synonymy and translation between modal logics [2], the authors attempted to show that there are two modal logics so that each is exactly translatable into the other, but they are not translationally equivalent. Unfortunately, there is an error in the proof of Theorem 4.7 of this paper, that purports to prove this result, as Lloyd Humberstone discovered [1].

In $\S 4$ of the published article, the authors define two modal logics, $\mathbf{T}^{*}$ and $\mathbf{K U}^{*}$, each of them formulated with a necessity operator $\square A$, together with a propositional constant $C$. Theorem 4.7 claims that there are translations $t_{1}$ and $t_{2}$ between the two logics, so that each logic is exactly embedded in the other, but that the two logics are not translationally equivalent. However, as Humberstone has pointed out, there is a fatal mistake in the proof that the translation $t_{2}$ embeds the logic $\mathbf{T}^{*}$ exactly in the logic $\mathbf{K} \mathbf{U}^{*}$. The formula

$$
C \rightarrow[\square(\square C \wedge C) \wedge \square C \wedge C]
$$

is provable in $\mathbf{K U}^{*}$ by the rule of necessitation and propositional logic; however, this formula is $t_{2}(C \rightarrow \square \square C)$, and this is not provable in $\mathbf{T}^{*}$, since in that logic no special axioms are assumed for the propositional constant $C$.

In the remainder of this article, we give an alternative (and hopefully correct!) proof of the same result, namely that there are two normal modal logics that are exactly translatable into each other, but not translationally equivalent. The two logics are defined by adapting a construction from [3].

\section{The new example}

We denote the set of non-negative integers by $\mathbb{N}$, and the set of positive integers by $\mathbb{N}^{+}$. The logics that we define in this section have a single necessity operator $\square$, and two constants $I$ and $E$ (they can be read as "initial point" and "end point"). Each logic $L_{X}$ is defined in terms of a set $X$ of positive integers.

Given $X \subseteq \mathbb{N}^{+}$, the logic $L_{X}$ is defined to be the smallest normal modal logic containing the following axioms:

1. $\diamond A \rightarrow \square A$; 
2. $\neg(I \wedge E)$;

3. $\neg \diamond I ;$

4. $I \rightarrow \neg \nabla^{k} E$, for $k \in X$.

That is to say, $L_{X}$ contains the usual axioms and rules for the smallest normal modal logic $K$, including the rule of Necessitation, together with the axioms listed above.

The notions of frame and model for $L_{X}$ are defined in the standard way. Thus, a frame for $L_{X}$ is of the form $\langle W, R, I, E\rangle$, where $W$ is a non-empty set, $R$ a binary relation on $W$, and $I, E \subseteq W$. The truth condition for a formula $\square A$, given an assignment of propositional variables to points in a frame, is as usual. The propositional constant $I$ is true at a point $x$ if and only if $I x$; similarly for the constant $E$.

Lemma 2.1 The logic $L_{X}$ is complete with respect to the class $C_{X}$ of frames satisfying the conditions:

C1: $(x R y \wedge x R z) \rightarrow y=z$;

C2: $\neg(I x \wedge E x)$;

C3: $x R y \rightarrow \neg I y$;

C4: $\left(I x \wedge x R^{k} y\right) \rightarrow \neg E y$, for $k \in X$.

Proof. Soundness and completeness are easily verified by the usual methods involving the canonical model.

The class of frames in the preceding Lemma can be described informally as follows. The accessibility relation is a partial function $f$ defined on the universe; $I$ and $E$ are disjoint subsets of the frame, where $I$ is a subset of the initial points (those points not in the range of $f$ ); there is no directed path of length $k$ from a point in $I$ to a point in $E$.

Lemma 2.2 Let $X, Y \subseteq \mathbb{N}^{+}$satisfy the condition

$$
\forall k \in \mathbb{N}^{+}(k \in X \Longleftrightarrow 2 k \in Y) .
$$

Then there is an exact translation from $L_{X}$ into $L_{Y}$ given by:

$$
t: \square \alpha \longmapsto \square \square \alpha ; I \longmapsto I ; E \longmapsto E .
$$

Proof. The fact that the translation is sound with respect to the axioms and rules of $L_{X}$ follows easily from the definition of $L_{X}$ and $L_{Y}$.

For the converse, let $A$ be a non-theorem of $L_{X}$. By Lemma 2.1, there is a frame $\mathcal{F}=\langle W, R, I, E\rangle$ in $C_{X}$, and a point $w \in W$ so that the formula $A$ is false at $w$, with respect to an assignment $\varphi$ of propositional variables to points in the frame. Define a new frame as follows. For each pair $\langle x, y\rangle$ in $R$, let $\beta_{x y}$ 


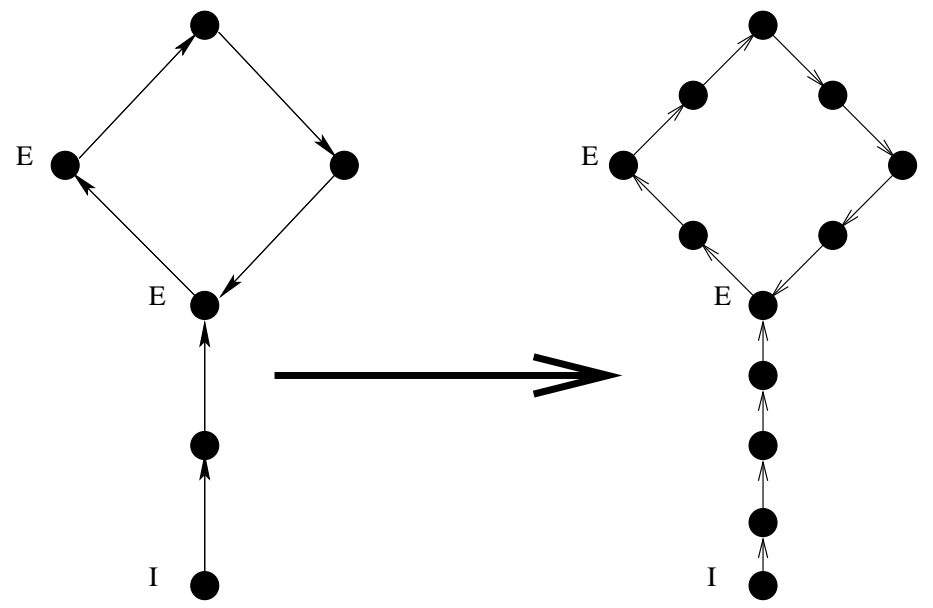

Figure 1: Construction of $\mathcal{F}^{*}$ from $\mathcal{F}$

be a new point; let $W^{*}$ be the set of all such new points. Define the accessibility relation on $W \cup W^{*}$ to be

$$
R^{*}=\left\{\left\langle x, \beta_{x y}\right\rangle,\left\langle\beta_{x y}, y\right\rangle:\langle x, y\rangle \in R\right\}
$$

that is to say, we insert the intermediate element $\beta_{x y}$ between the points $x$ and $y$ in the original frame. The new frame is then $\mathcal{F}^{*}=\left\langle W \cup W^{*}, R^{*}, I, E\right\rangle$, and in addition, the assignment $\varphi$ is extended to the new frame by setting all propositional variables to 0 at all of the new points. The construction is illustrated in Figure 1.

If $x, y \in W$, then $x R y$ holds if and only if $x\left(R^{*}\right)^{2} y$. From this fact, it can be proved by induction on the complexity of a formula $B$ that $B$ is true at a point $x \in W$ in the original model based on the frame $\mathcal{F}$ if and only if $B^{t}$ is true at $x \in W$ in the new model based on the frame $\mathcal{F}^{*}$. Consequently, $A^{t}$ is false at $w$ in the new model. It remains to show that the new frame $\mathcal{F}^{*}$ belongs to $C_{Y}$. It is easy to see that the frame $\mathcal{F}^{*}$ satisfies the first three conditions of Lemma 2.1. To prove the fourth condition, assume that it fails for some $k \in Y$. Hence, for some $x, y, I x \wedge x\left(R^{*}\right)^{k} y \wedge E y$, for $k \in Y$. Since $x, y \in W$, it follows that $k$ is even, so that $k=2 m$ for some $m \in X$, showing that $x R^{m} y$ for some $y \in E$. This contradicts the assumption that the original frame $\mathcal{F}$ is in $C_{X}$.

Theorem 2.1 Let $X=\left\{4^{k}: k \in \mathbb{N}\right\}$, and $Y=\left\{2 \cdot 4^{k}: k \in \mathbb{N}\right\}$. Then the logics $L_{1}=L_{X}$ and $L_{2}=L_{Y}$ are not translationally equivalent, although there are exact translations from $L_{1}$ into $L_{2}$ and from $L_{2}$ into $L_{1}$.

Proof. The condition of Lemma 2.2 is satisfied by $X$ and $Y$, and conversely by $Y$ and $X$. Consequently, Lemma 2.2 implies that there are exact translations in either directions between the logics. 
It remains to be shown that the logics are not translationally equivalent. To do this, we shall enumerate for each logic the number of two-element frames that validate it. There are exactly six non-isomorphic partial functions on the two-element set $W=\{1,2\}$. We consider these in order. Our procedure in each case is to consider all of the frames based on a given partial function that satisfy the first three conditions of Lemma 2.1, C1 to C3. This means choosing $I, E \subseteq\{1,2\}$ so that $I$ is disjoint from both $E$ and the range of the partial function. We then examine the frame further to see if it satisfies the fourth condition, C4, for each of the two logics.

Case A: $R=\emptyset$. Then there six non-isomorphic frames satisfying $\mathbf{C 1}$ to $\mathbf{C 3}$. All six of them validate both logics (trivially, since the relation is empty).

Case B: $R=\{\langle 1,1\rangle\}$. Then there are again six non-isomorphic frames satisfying $\mathbf{C 1}$ to $\mathbf{C 3}$. Again, all six validate both logics, because $I$ and $E$ must be disjoint.

Case C: $R=\{\langle 1,1\rangle,\langle 2,2\rangle\}$. There are three non-isomorphic frames satisfying $\mathbf{C 1}$ to $\mathbf{C 3}$. All three validate both logics, because $I=\emptyset$.

Case D: $R=\{\langle 1,2\rangle,\langle 2,2\rangle\}$. There are six non-isomorphic frames satisfying C1 to C3. The first five validate both logics. The sixth (with $I=\{1\}$ and $E=\{2\})$ validates neither logic.

Case E: $R=\{\langle 1,2\rangle,\langle 2,1\rangle\}$. There are three non-isomorphic frames satisfying $\mathbf{C 1}$ to $\mathbf{C 3}$, all of which satisfy both logics, because in this case $I=\emptyset$.

Case $\mathbf{F}: R=\{\langle 1,2\rangle\}$. There are six non-isomorphic frames satisfying $\mathbf{C 1}$ to C3. Of these, the first five satisfy both logics, but the last, with $I=\{1\}$ and $E=\{2\}$, validates $L_{2}$, but not $L_{1}$, since $1 \in X, 1 \notin Y$.

To sum up, there are 29 non-isomorphic 2-element frames validating $L_{2}$, but only 28 non-isomorphic 2-element frames validating $L_{1}$. Hence, by Theorem 4.3 of [2], $L_{1}$ and $L_{2}$ are not translationally equivalent.

The logics in our new example use two propositional constants, in addition to a modal operator. It would be interesting to eliminate the constants, and construct two normal modal logics with the same property, but having a oneplace modal operator as the sole non-classical connective.

We wish to express our thanks to Lloyd Humberstone for calling our attention to the error in our paper, and also for useful comments on an earlier draft of this note. Thanks also to Allen Hazen for interesting comments about problems of translation in modal logics.

Lloyd Humberston also called our attention to a significant paper by Evgeni Zolin [4] that we overlooked in the references to our article. In that article, Zolin considers the problem of exact translations from one modal logic into another, and proves some surprising negative results, including the impossiblity of embedding certain strong modal logics like $S 5$ into weak logics such as $K$.

\section{References}

[1] Lloyd Humberstone. A problem with Theorem 4.7 of 'Synonymous Logics'. One page manuscript, August 2006. 
[2] Francis Jeffry Pelletier and Alasdair Urquhart. Synonymous logics. Journal of Philosophical Logic, 32:259-285, 2003.

[3] Alasdair Urquhart. Decidability and the finite model property. Journal of Philsophical Logic, 10:367-370, 1981.

[4] Evgeni E. Zolin. Embeddings of propositional monomodal logics. Logic Journal of the IGPL, 8:861-882, 2000.

Simon Fraser University

Burnaby, British Columbia

University of Toronto

Toronto, Ontario 